\title{
La revitalización del Centro Histórico de Zacatecas y la conciencia social
}

\section{The revitalisation the Historic Centre of Zacatecas and social conscience}

\author{
Guadalupe M argarita González-H ernández*
}

\begin{abstract}
The historic centre of the city of Zacatecas (M exico) has been transformed in the ideal place for tourists and high-income consumers through the usage of a colonial quarter that, as a part of cultural heritage, has imposed rehabilitation policies. In parallel, a suburbanisation and conurbation process towards the neighbouring city of Guadal upe has generated a spatial segmentation by increasing displacement costs. The historic centre of Zacatecas is nowadays a public space with private usage that is thought of as being visually stunning, ideal for freetime activities, leaving behind its image as a place where cl ass struggl es converge.
\end{abstract}

Keywords $\mathrm{H}$ istoric Centre, transmutation, social conscience, suburbanisation.

Resumen

El Centro H istórico de la ciudad de Zacatecas (M éxico) se ha convertido en el lugar ideal para turistas y consumidores de altos ingresos, mediante el aprovechamiento de un casco colonial que, en tanto patrimonio cultural, obliga a implantar una política de rehabilitación. Paralelo a ello, el proceso de suburbanización y conurbación hacia la vecina ciudad de Guadalupe ha generado una segmentación espacial, al incrementarse los costos de desplazamiento. Actualmente el Centro $\mathrm{H}$ istórico de Zacatecas es un espacio público con uso privado que se concibe como estéticamente espectacular, idóneo para actividades de ocio, dejando atrás su imagen como un lugar donde convergen las luchas de clases.

Palabras clave: Centro H istórico, transmutación, conciencia social, suburbanización.

* Universidad Autónoma de Zacatecas, M éxico. Correo-e: gmgonzh@ uaz.edu.mx. 


\section{Introducción}

Las remodelaciones que se Ilevaron a cabo en el Centro $\mathrm{H}$ istórico de la ciudad de Z acatecas ( $\mathrm{CHz}$ ) en los últimos años coinciden más con el proyecto neoliberal de hacer agradable un casco colonial para los turistas y los consumidores de altos ingresos, quienes fomentan actividades - muy lucrativas- de ocio, que con crear un espacio público donde se reproduzcan las prácticas cotidianas sociales. Se trata de un proceso en el que la idea de protección del patrimonio cultural sirve para usar un espacio público con fines económicos privados sin que los pobladores lo perciban de manera clara.

Al mismo tiempo que se llevan a cabo las obras de revitalización del Centro Histórico, en la ciudad se desarrollan dos procesos de forma tardía y subdesarrollada: la suburbanización y la conurbación con la vecina Villa de Guadalupe, los cuales, en cierto modo, han ocultado los intereses de clase creados.

La expansión de la ciudad de Z acatecas hacia Villa de Guadalupe se caracteriza principalmente por, primero, el desplazamiento de pobladores de ingresos altos y medios hacia la zona $\mathrm{Cam}$ pestre-Bernárdez provocado por políticas de fomento a la vivienda residencial y semirresidencial donde los capitales inmobiliarios y de construcción cumplieron un papel fundamental; segundo, una política de creación de infraestructura (basada casi exclusivamente en complejos viales) que sustenta la expansión urbana y permite el traslado diario de la población a sus centros de empleo, comercios y servicios en medios de transporte privados; y por último, una política de revitalización del Centro $\mathrm{H}$ istórico de la ciudad de Zacatecas ( $\mathrm{cHz}$ ) Ilevado a cabo por el Estado y alentado por el capital local y nacional, desplazando las actividades no centrales (comercio al por mayor, actividades artesanales y manufactureras) y a pobladores de bajos ingresos hacia la periferia mediante mecanismos formales (dotación de vivienda de interés social) y no formales (invasión de terrenos). Las consecuencias son una clara segregación socioespacial en beneficio de las clases con mayor poder económico y político, así como el fomento, casi exclusivamente, de la actividad turística que, aunque no tiene un efecto económico importante en la ciudad, sí modifica las percepciones sobre el uso y fines del $\mathrm{CHz}$. 
Para fundamentar dicha aseveración utilizamos datos económicos del $\mathrm{CHz},{ }^{1}$ y también se identificaron procesos de estructura de centros ${ }^{2}$ para tres periodos (1990-1993, 1995-1998 y 20002003) con el fin de resaltar la importancia del CHz en la economía de la ciudad Zacatecas-Guadalupe (czG). Además se aplicó una encuesta a visitantes del $\mathrm{CHz}$ (turistas, visitantes regionales y pobladores de la ciudad) y finalmente se entrevistó a funcionarios de dependencias turísticas para conocer sus percepciones acerca del $\mathrm{CHz}$.

Este artículo consta de ocho apartados. El primero se refiere al debate sobre las tendencias teóricas de los estudios sobre centros históricos. El segundo trata acerca de nuestra concepción sobre la centralidad y la especificidad de aquellos centros históricos donde se expresan las prácticas sociales. En el tercer apartado se expone el papel de la czg en su dinámica regional. En el cuarto se habla de los cambios de la czG en su estructura urbana en la década de los noventa y cómo se ha beneficiado el CHz. El quinto presenta la evolución del proceso de revitalización y el papel del Estado en su éxito. El sexto plantea la importancia del turismo en la economía de la czG. El séptimo muestra el efecto espacial que ha tenido el turismo en el cHz y expone cómo se convirtió en un espacio público de uso privado que cambió la percepción de un lugar donde se resolvían los conflictos sociales al espacio dedicado al ocio y donde se disfruta la belleza estética. Finalmente se dan las conclusiones.

\section{Tendencias y diferencias teóricas}

Los estudios sobre la decadencia del distrito central de negocios (DCN), donde se mostraba su desaparición y el surgimiento de otros centros basados principalmente en negocios financieros, fueron el punto de partida para el ingreso de nuevas ideas y corrientes teóricas acerca de la función de los centros históricos y el papel de las políticas urbanísticas sobre su mantenimiento.

Las tendencias surgidas en Estados Unidos a comienzos de Ios noventa (Richardson y Gordon, 1994; Gordon y Richardson, 1995, 1995a y 1996) planteaban la desaparición de loS DCN a pesar del impulso de regenerarlos y modernizarlos, debido a que había, primero, otras áreas que ofrecían bienes y servicios más

\footnotetext{
${ }^{1}$ Tomados de los censos económicos del IN EGI (1989, 1994, 1999 y 2004).

${ }^{2}$ Con técnica de Alegría (1994a: 42-43) en área geoestadística básica (AGEB), utilizando datos económicos y de población del INEGI (1992, 1994, 1996, 1999, 2000 y 2004).
} 
competitivos y, segundo, loS DCN habían sido poblados por grupos étnicos segregados que los convirtieron en su área de control. Las políticas de remodelación y regeneración de loS $D_{C N}{ }^{3}$ fueron altamente segregadoras pues los repoblaron con grupos de altos ingresos, que demandan ciertos bienes y servicios (moda, entretenimiento y cultura) expulsando a quienes no cumplían con esos requisitos (grupos étnicos, familias) (0 'Sullivan, 1996).

Desde aquellos primeros estudios se ha cambiado y redirigido el análisis del centro histórico: la idea de su desaparición decayó (salvo excepciones como en Los Ángeles, que es el prototipo de una ciudad descentralizada). Incluso en Estados Unidos se ha escrito que los centros históricos se pueden seguir manteniendo mediante la especialización (Kotkin, 1999; Staley, 2004), ya no digamos en Europa y Latinoamérica, donde los centros históricos tuvieron un estado de decadencia menor que aquellos (Rossi, 2003).

El nivel de desarrollo y el papel que juega la dinámica internacional es un factor que se debe considerar. El grado de deterioro del centro histórico depende del papel que cumple la organización económica y política en el ámbito mundial. Las ciudades de Estados Unidos han sufrido fuertemente los procesos mundiales de relocalización productiva. Ante la constante búsqueda de reducción de costos de transporte y mano de obra, la mayor parte de la industria ubicada en loS DCN buscó nuevos lugares que le proporcionaron mayor rentabilidad, como en el caso de las ciudades latinoamericanas en un primer momento (durante los sesenta y setenta) y posteriormente las ciudades asiáticas.

Además, el deterioro de loS DCN fue mayor debido al papel que cumplió el espacio y las rentas del suelo, cuyo costo permitió que aquellas actividades económicas que no podían costearlas, buscaran la manera de atraer población hacia los suburbios o hacia áreas con rentas del suelo más baratas aprovechando la proliferación de tecnologías de comunicación (telefonía, fax, computadora y la Internet) y transporte (automóviles y camiones). Las actividades dominantes mundialmente abandonaron el DCN, el cual fue ocupado por actividades y pobladores que no tenían relevancia de acuerdo con la dinámica capitalista (comercio ilegal y grupos étnicos, entre otros).

Con el fin de devolverle su esplendor, los gobiernos locales regeneraron el DCN en dos sentidos: a) repoblarlo, restaurar sus

${ }^{3}$ En Estados Unidos les Ilaman políticas de gentrification y en Europa de elitización. 
edificios o construir nuevos para convertirlos en departamentos habitacionales y oficinas para actividades que requieren de contacto cara a cara; los empleados de actividades como la moda, la cultura y el entretenimiento serían quienes le darían nueva vida; y b) especializarlo, dinamizar su economía con base en actividades que requieren un contacto cara a cara o que permiten convertirlo en zona de entretenimiento o de cultura (principalmente posmodernista). En términos analíticos, el DCN seguirá siendo un área importante dependiendo del grado de desarrollo y especialización que incentive su economía.

A pesar de los esfuerzos, las políticas de regeneración tuvieron consecuencias poco exitosas en el repoblamiento, pues la llegada de jóvenes solteros con altos ingresos no contribuyó a que aumentara su demografía, y en la mezcla social, debido a la expulsión hacia otras áreas de grupos étnicos por jóvenes blancos con nivel de vida alto (O 'Sullivan, 1996).

Aunque en Europa se dio esta tendencia, el resultado es diferente. El centro histórico cumplió otras funciones que impidieron su deterioro tanto como en las ciudades estadounidenses. Un factor importante es el turismo. Desde hace tiempo las ciudades europeas son uno de los destinos preferidos por este sector, lo que permite que actividades de ocio (entretenimiento, moda, cultura, gastronomía) busquen el centro histórico como su objetivo de ubicación, y de esta manera mantenerlo y conservarlo. O tro factor es el espacio y la renta de suelo. La disputa por el suelo es mayor en Europa y por tanto su costo se ha incrementado. Ante esta situación, las actividades económicas no tienen motivos suficientes para desplazarse fuera del centro histórico; sin embargo, el exceso de actividades en estos sitios ha traído costos de aglomeración (congestionamento vial, contaminación, etc.) que lo han deteriorado, aun cuando se le dé mantenimiento (R ossi, 2003). N o obstante, estas políticas implican que, en aras de aprovechar la actividad turística, se expulsen grupos sociales que deslucen el casco central. En las ciudades europeas se hizo lo necesario para que el centro histórico sea un lugar chic, cultural y asocial (O rtiz, 2005).

Al igual que en Estados Unidos, sólo las ciudades con presencia mundial llevaron el proceso de manera rápida y de acuerdo con la dinámica mundial; el resto mostró síntomas y ritmos diferentes (Gamarra, 2005).

Desde los años sesenta y hasta los ochenta las ciudades latinoamericanas se convirtieron en el lugar por excelencia de loca- 
lización del capital internacional, ya que en ellas se encontraba abundante mano de obra barata (que creció con las migraciones del campo a la ciudad), privilegios fiscales y recursos naturales sin control legal. Posteriormente, ante el cambio en las condiciones económicas internacionales (el proceso de reestructuración productiva), estas ciudades fueron abandonadas por el capital transnacional para irse a Asia, lo que intensificó problemas de sobrepoblación ampliada y de acumulación. Las ciudades tuvieron que reestructurarse.

En Latinoamérica, a diferencia de Europa, el espacio no es un problema, pero sí la restricción de ingresos. Por su condición de región subdesarrollada, la población y las actividades económicas no han tenido los recursos suficientes para ubicarse en la periferia ni para acceder a la última tecnología en comunicación y transporte. Por ello, las clases sociales altas fueron quienes primero poblaron el centro histórico y después convivieron con los cinturones de miseria en la periferia (Jaramillo, 1997), con la característica de que al convivir y distinguirse de los que no eran de su condición, se autosegregaron en colonias amuralladas (ThuiIlier, 2005). Al igual que las ciudades europeas, sólo las más importantes de Latinoamérica (ciudad de M éxico, Santiago, Río de Janeiro, Buenos Aires) mostraron tendencias a la descentralización en forma relevante, el resto tuvo ritmos y fases distintas de desarrollo urbano y económico.

Ante las diferencias, los estudios urbanos han identificado que no es posible seguir las tendencias estadounidenses como modelo; por el contrario, es necesario hacer un estudio detallado con el fin de encontrar las especificidades y, si existen generalidades, ponerlas en evidencia. En la actualidad los casos específicos han permitido identificar dos tipos de estudios: a) quienes plantean que el centro histórico se remodeló y recobró su brillo gracias a los planes maestros de urbanismo gestionado por el Estado, financiado por el capital y ejecutado con la participación ciudadana (Rossi, 2003; Carrión, 2005), y b) aquellos que muestran que el centro histórico presenta una revitalización inducida por el capital y gestionada por el Estado, es decir, una política de regeneración y/o remodelación agradable para las clases altas (Gamarra, 2005; H iernaux, 2005; Low, 2005).

El análisis del centro histórico no se debe separar del estudio de la suburbanización, o por lo menos así debe ser para las ciudades que no cumplen con lo que requiere la dinámica capitalista internacional. M ientras que en las ciudades donde dominan 
las innovaciones en el proceso productivo (Los Ángeles, Tokio, Sydney, N ueva York) la suburbanización se dio antes que la reestructuración, en las ciudades subdesarrolladas la suburbanización se traslapa con el proceso de revitalización de la ciudad. N o es contradictorio, simplemente son ritmos diferentes y tardíos.

\section{Desde los centros de comercio y servicios hasta los centros de prácticas sociales}

Los centros se han definido de acuerdo con la posición teórica y la disciplina que los estudie. D esde las investigaciones económicas que definen principalmente a un centro como la concentración de actividades económicas establecidas en un lugar específico aprovechando las economías de escala, ${ }^{4}$ de aglomeración y de alcance ${ }^{5}$ (González, 2004: 30), hasta los estudios antropológicos que definen a los centros como lugares donde los imaginarios de prácticas social es se expresan de manera dominante (H iernaux, 2005).

Consideramos los centros como sitios de servicios y comercios que se originan como lugares donde se concentra la mayor cantidad de actividades de servicios o comerciales que abastecen un área de mercado o zona de influencia contigua. Se expresan en el espacio urbano como centros de servicios y comercios en ciudades predominantemente terciarias. Se ubican en el territorio urbano de manera espaciada y de modo jerárquico en sus funciones. Los mecanismos de mercado provocan que "Ios centros de mayor jerarquía estén asociados con las zonas donde se localizan los grupos de altos y medios ingresos" (Alegría, 1994a: 21). El fuerte poder de compra de los grupos de ingresos altos y medios permite mayor cantidad, diversidad y frecuencia de productos (González, 2004 y 2006). El acceso y la densidad del empleo, así como la infraestructura se asocian espacialmente a la localización de los centros de servicios y comercios (Alegría, 1994). En general, en las ciudades tradicionales mexicanas los centros de mayor envergadura se ubican en el mismo espacio del casco histórico, lo que crea una simbiosis entre centro y centro histórico.

En términos simbólicos, los centros son el lugar donde confluyen todas las prácticas sociales cotidianas, es donde se expre-

\footnotetext{
${ }^{4}$ El incremento del tamaño productivo de un negocio debido al aumento de las unidades de algún factor de producción (capital o trabajo) (González, 2004).

${ }^{5} \mathrm{~L}$ a capacidad productiva de los negocios para ofrecer gran variedad de servicios 0 de bienes.
} 
san las contradicciones individuales y colectivas de los habitantes de las ciudades; aunque en las ciudades globales los centros han dejado de ser expresión de los imaginarios urbanos (H iernaux, 2005: 19).

D esde nuestra perspectiva, los centros históricos se han convertido en los lugares idóneos donde se aprovechan ciertos imaginarios urbanos y los espacios públicos para fines privados. Se trata de reelaborar una conciencia social ${ }^{6}$ que proteja el casco histórico para hacer rentables actividades de ocio o entretenimiento. El centro histórico dejó de ser el sitio donde divergen o convergen las luchas de clases para convertirse en un lugar donde se disfruta de un patrimonio cultural que nos ofrece artículos suntuarios, sobre todo en ciudades donde es parte fundamental de su estructura económica. Existe un proceso de transmutación de la conciencia social que acepta comercializar un centro histórico y desterrar a quienes no coinciden con sus intereses económicos. El motivo más importante es el económico, aunque que disfraza de orgullo por lo estético, lo histórico y lo cultural.

\section{La ciudad $Z$ acatecas-G uadalupe y su papel en la región}

Ésta se conforma con la unión espacial de la capital del estado de Zacatecas y su vecina Villa de G uadalupe. Localizada en la parte centro-norte del país, su economía se fundamenta en la administración pública, así como en los servicios educativos, personales y turísticos ${ }^{7}$ (INEGI, 2006). De tamaño medio ${ }^{8}$ y con origen minero colonial, la czg ha dependido del gasto público que se derrama en la actividad local mediante recursos destinados a la actividad primaria, ${ }^{9}$ infraestructura básica, servicios urbanos y para sustentar una política social (cada vez más restringida por un Estado federal neoliberal) "cuyo objetivo no es más que el de

\footnotetext{
${ }^{6}$ Percepciones, ideas, imaginarios sobre las diferencias sociales e intereses de clases que se modifican de acuerdo con las dinámicas sociales, económicas y políticas en un contexto definido en tiempo y espacio.

7 De acuerdo con la Encuesta $\mathrm{N}$ acional de Empleo Urbano (enEu), 39.4\% de la población ocupada en la czG en el año 2004 se encontraba en la rama de servicios, 23\% en el comercio, $12.9 \%$ en el gobierno (federal, estatal o municipal), $9.4 \%$ en la industria de la transformación, $8.7 \%$ en la industria de la construcción, $4.3 \%$ en comunicaciones y transportes, $1.3 \%$ en actividades primarias y, finalmente, $1 \%$ en la industria extractiva y eléctrica. Este comportamiento se mantiene desde 1992 (INEGI, 2006).

${ }^{8}$ Según el II Conteo 2005, la czG cuenta con 222,461 habitantes (IN EGI, 2006a).

${ }^{9}$ Las aportaciones y subsidios para estimular la producción que obtienen campesinos y pequeños productores del estado de programas como Procampo, los acapara la CZG ya sea en la compra de herramientas o insumos, pues es la capital quien controla el comercio regional.
} 
aminorar los efectos del escaso desarrollo económico prevaleciente" (Esparza, 1996: 43).

Las relaciones sociales capitalistas de producción no se establecen como las dominantes, ya que la relación capital-trabajo es marginal. El empleo asalariado no lo representa el proletariado sino los burócratas gubernamental es y los trabajadores de la educación ${ }^{10}$ (Delgado et al., 1991: 33-34). El capital sólo se maneja en pequeña escala (principalmente industria de la construcción y comercio local) o por grandes cadenas transnacionales (Wal M art, Sam's Club) o nacionales (Soriana) que controlan el comercio local. Por tanto, la población que no está en el trabajo asalariado o en el sector empresarial simplemente es sobrepoblación absoluta, es decir, población redundante o excedente que vive en condiciones de subsistencia y cuya única válvula de escape es la emigración o el trabajo subsidiario a la acumulación: trabajo por cuenta propia, a destajo, no remunerado, subcontratación, entre otros (Delgado et al., 1991; Figueroa, 1986).

\section{C ambios en la estructura de centros en la ciudad $Z$ acatecas- G uadalupe}

Como la gran mayoría de las ciudades mexicanas, la czg ha cambiado en su estructura urbana, los procesos económicos, demográficos y sociales han determinado una dinámica que se resume de la siguiente manera.

1. En la czG los procesos de reestructuración, ${ }^{11}$ suburbanización y revitalización del Centro Histórico han sido casi simultáneos, en contraposición con la tendencia estadounidense de evolucionar desde la suburbanización hacia la descentralización económica y luego a la revitalización del distrito central de negocios (González, 2004, 2006, 2006b). La suburbanización incluye a todos los estratos sociales en fases diferenciadas, pero destaca que los grupos sociales de menores ingresos se trasladaron hacia las zonas periféricas en virtud de los costos de la tierra y de la construcción (González et al., 2007), en tanto que los grupos de

${ }^{10}$ Con base en la Encuesta $\mathrm{N}$ acional de Empleo (INEGI, 2005), en el último trimestre de 2004 la población ocupada asalariada estuvo contratada en servicios educativos y sociales (40\%), comercio (39\%) y en la administración pública $(21 \%)$.

${ }^{11}$ Es decir, la modificación en las concentraciones de actividad económica en los distintos espacios urbanos y en la jerarquía que al gunas de estas áreas ejercen sobre las otras. 
ingresos medios y altos se asentaron en áreas a las que se dotó de infraestructura urbana que incluyó accesos viales y espacios para su explotación comercial (plazas y centros comerciales). Por tanto, las clases de altos ingresos pueden realizar cerca de su domicilio buena parte de sus actividades de compra y consumo, mientras que las poblaciones de menores ingresos al no recibir equipamiento urbano, vial o comercial cerca de sus asentamientos debe efectuar dichas actividades en establecimientos que permitan la ventaja de la aglomeración (González, 2004, 2006).

2. La czg reestructuró sus centros en tres periodos relativamente cortos (1990-1993, 1995-1998 y 2000-2003) en los que se evidencia, en el primer periodo, cómo transita de una estructura monocéntrica (en la que coinciden el centro principal con la cHz) hacia una estructura policéntrica (donde se configura la extensión del centro principal hacia las AGEB, Área Geoestadística Básica, colindantes con el cHz) para el periodo 1995-1998. En las figuras । y II se da cuenta de esta primera transformación. La aparición de nuevos subcentros en áreas a las que se dotó de equipamiento urbano y vocación comercial en torno a los cuales se asentaron grupos de medios y altos ingresos, así como un crecimiento de la actividad de servicios al turismo explican esta transformación (González, 2004). Finalmente, en el periodo 2000-2003 la czg retoma su carácter monocéntrico, con lo que refuerza la identificación entre $\mathrm{CHz}$ y centro principal. La mayor concentración de actividades económicas en el casco histórico con respecto a otras áreas de la czg se puede explicar por la conjunción de varios factores: pérdida de población; cambio de uso de los edificios localizados en el $\mathrm{CHz}$, de habitacionales a comerciales y de servicios; incremento del personal ocupado en sus establecimientos y, sobre todo, el fortalecimiento de las ventajas que otorgan las economías de escala: alcance y aglomeración. Es decir, el chz recuperó su competitividad como área geográfica atractiva para las actividades económicas que cubren las demandas de la población local, pues en ese periodo las actividades relacionadas con el turismo se encontraban estancadas (figura III). Asimismo es posible afirmar que los bienes y servicios producidos en el área geográfica de nuestro estudio no cubren las necesidades básicas, sino que están dirigidos a mercados 


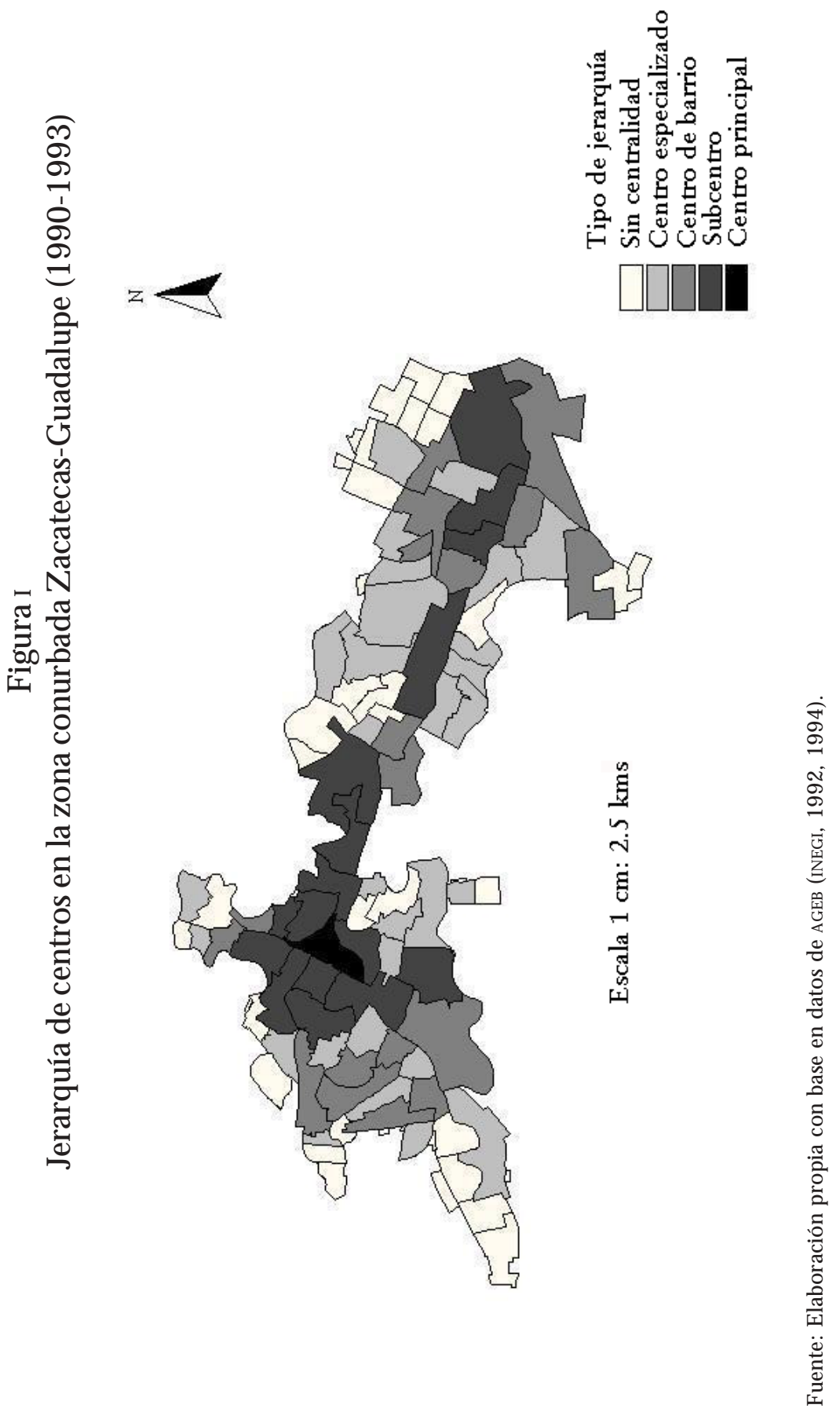




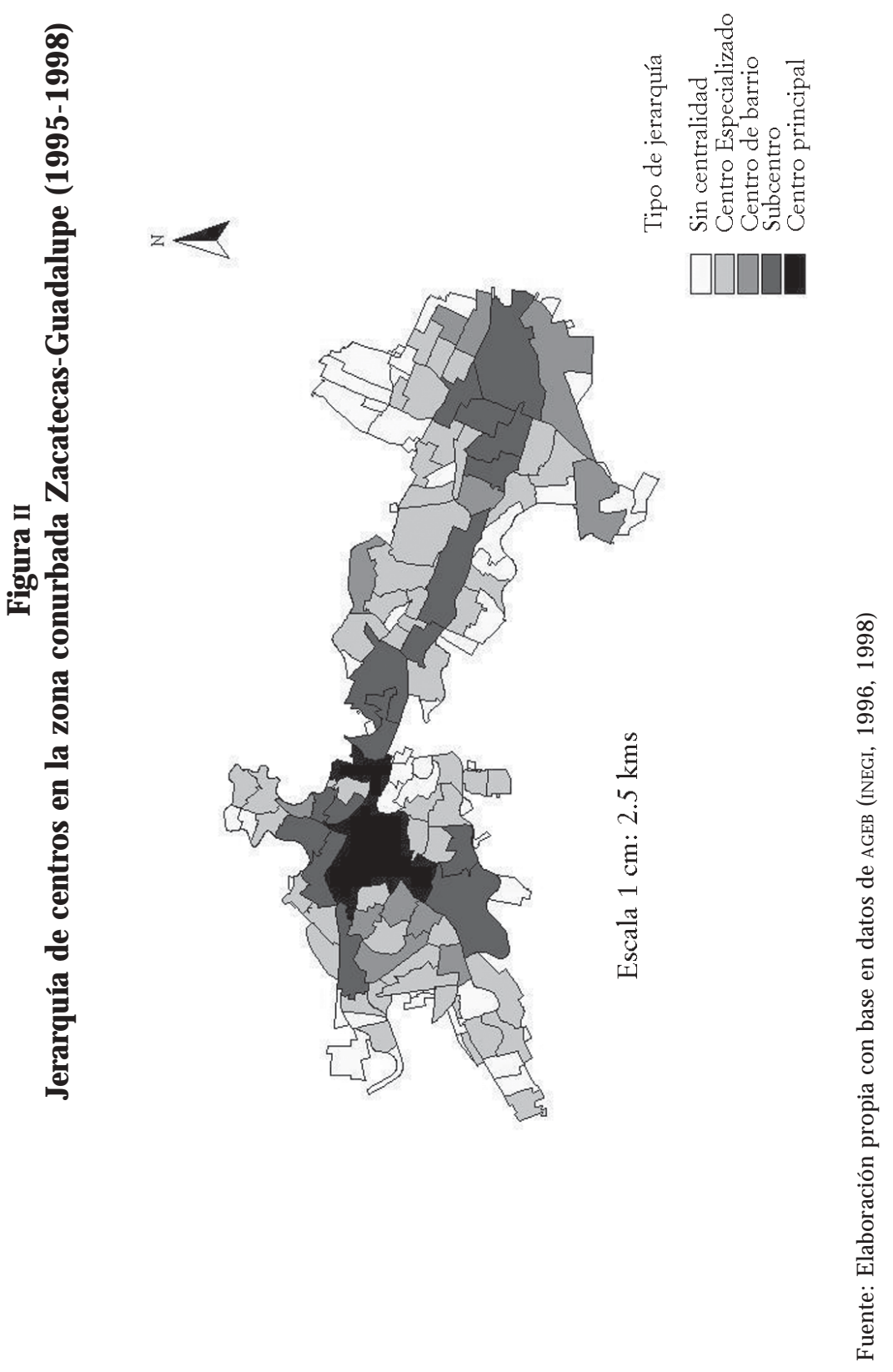


de ingresos medios y altos, pues los establecimientos corresponden a giros de entretenimiento y venta de productos especiales como regalos, teléfonos, moda y muebles, o bien a servicios de restaurantes, cafeterías, bares y antros (González, 2006).

3. Al consolidarse la actividad económica en el cHz a comienzos del siglo xxı, los costos de desplazamiento se incrementaron debido a que la población ya vive en la periferia y la actividad económica se encuentra sobre todo en dicho centro. Es decir, la expansión y conurbación de la ciudad significó un aumento en la distancia promedio que recorre buena parte de la población para realizar sus actividades diarias de trabajo, estudio u ocio. Este fenómeno implica dos cosas: la primera se refiere a un mayor gasto para desplazarse, en especial para quienes viven en la periferia de Guadalupe y llevan a cabo sus actividades en el $\mathrm{CHz}$, lo que les significa un problema de tiempo, dinero y esfuerzo; la segunda tiene que ver con las repercusiones que tiene ese desembolso en la distribución del gasto de los hogares (G onzález, 2006). Lógicamente, las familias de menor ingreso sacrifican satisfactores importantes (educación, vestido, entretenimiento) para solventar los costos y tiempos de transporte (G onzález et al., 2007). En síntesis, quienes tienen menor ingreso y viven en la periferia tienen un mayor costo de desplazamiento y peor distribución del gasto que aquellos que viven cerca del $\mathrm{CHz}$ y cuentan con salarios más altos. El deterioro del ingreso de la población se ha dado conforme se expande la ciudad (González, 2004, 2006). La característica principal del periodo 1990-1993 fue que quienes viven cerca del $\mathrm{cHz}$ gastaron notablemente menos en transporte en comparación con los pobladores de las periferias, quienes tuvieron menor capacidad de compra porque tienen costos de desplazamiento más al tos. Para el periodo 1995-1998 la tendencia siguió siendo la misma, pero la intensidad fue mayor. El ingreso se deterioró más al aumentar el número de centros, pues, como ya se mencionó, los grupos de ingreso alto y medio se localizan cerca de ellos. Para ejemplificar bastaría conocer que la diferencia entre el costo promedio de desplazamiento entre los grupos de ingresos bajo y alto fue de 19.7 veces. Durante este lapso la población con menores ingresos sufrió 


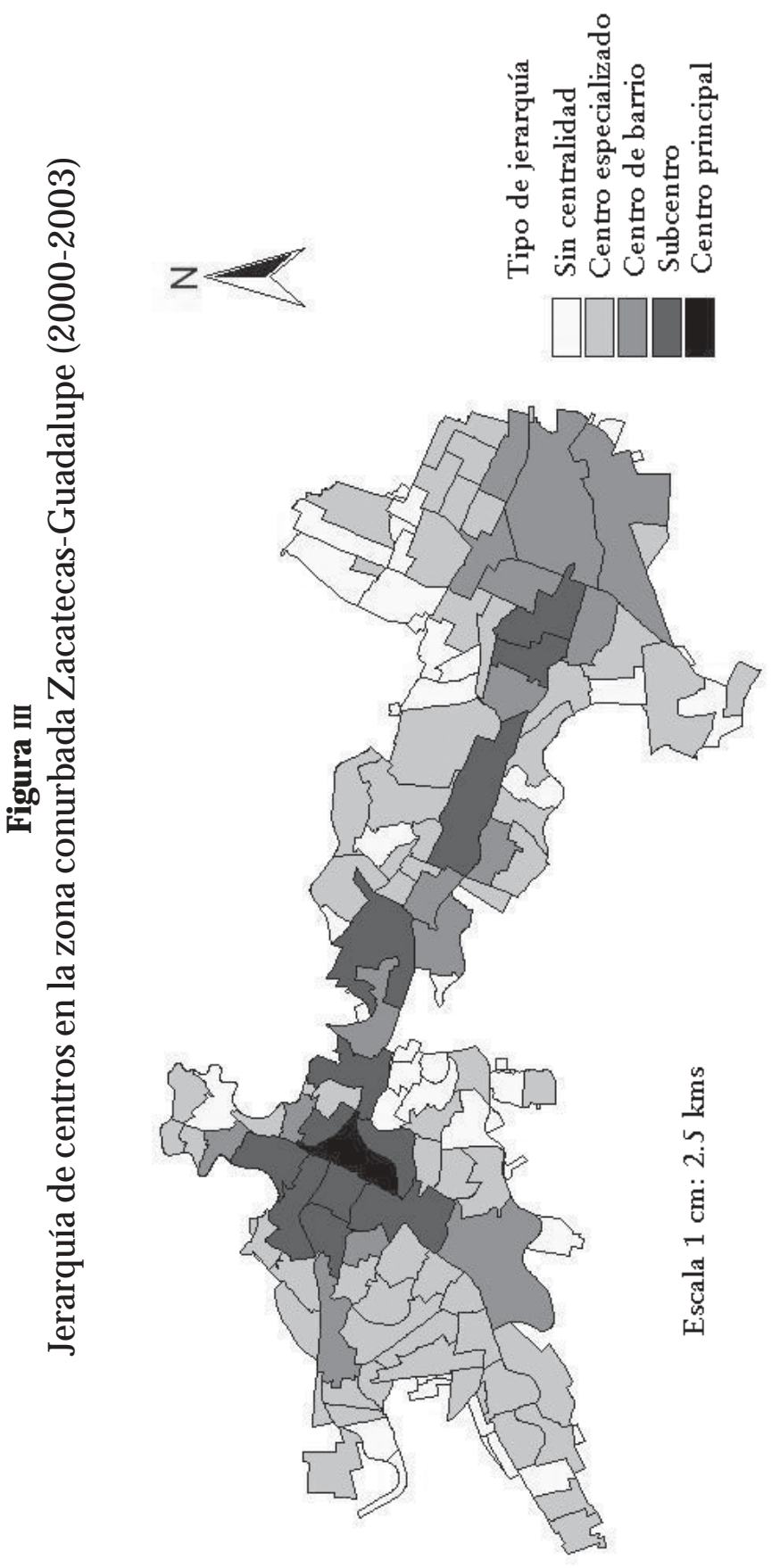

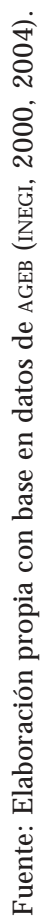


efectos muy negativos (González, 2004). Por último, en el periodo 2000-2003 el desarrollo del CHz en cuanto a comercios y servicios tuvo consecuencias contradictorias: por una parte, permitió disminuir el costo de desplazamiento de la población cercana al $\mathrm{CHz}$ y de los grupos de ingresos altos y medios que tienen la posibilidad de trasladarse sin inconvenientes hacia las áreas comerciales y de servicios, así como a sus centros de trabajo; y por otra, los habitantes de áreas lejanas a éste (en general de menores ingresos) incrementaron su costo de desplazamiento, lo que afecta sus patrones de consumo. Así, se puede afirmar que los fenómenos de reestructuración y suburbanización de la czG contribuyeron al deterioro espacial del ingreso de la población con menores recursos (G onzález, 2006, 2006a).

\section{El C entro $\mathrm{H}$ istórico de $\mathrm{Z}$ acatecas y sus políticas de revitalización}

Como vimos, en la czg no hay una diferencia clara en los tiempos históricos; por el contrario, la ciudad parece traslapar varias etapas históricas y solapar sus contradicciones inherentes.

En la czG los espacios públicos, aquellos lugares donde pueden estar y expresar sus ideas e intereses todos los individuos, se reducen a pequeñas plazas situadas en lugares poco redituables para la actividad inmobiliaria y frecuentemente invadidos por intereses privados comerciales (bien en forma de ambulantaje ${ }^{12} 0$ extensión de negocios establecidos ${ }^{13}$ ). Ante la ausencia de dichos espacios, las calles se vuelven expresión e identidad de los zacatecanos.

Con capital estatal se decidió renovar el Centro Histórico, ${ }^{14}$ focus de la vida social, económica, política y simbólica de la ciu-

12 Venta de comida o productos chatarra.

${ }^{13}$ Colocación de mesas y sillas de cafeterías, restaurantes, así como estantes de recuerdos turísticos, dulces y joyería.

${ }^{14}$ Aunque en la czG existen dos centros históricos (de las ciudades de Zacatecas y de Guadalupe), hablaremos sólo del de la capital del estado de Zacatecas, por dos razones. La primera, porque su poder político y económico es mayor que el de Guadalupe y porque acapara más recursos financieros (municipales, estatales, federales e incluso internacionales) para su mantenimiento. Y la segunda, porque aunque la política neoliberal también se ha aplicado en el Centro $\mathrm{H}$ istórico de Guadalupe, la transmutación de la conciencia social es mucho más evidente en la ciudad de Zacatecas que en Guadalupe, debido a que esta ciudad se ha visto afectada por diversos factores que hemos expuesto en otros espacios (González, 2004, 2006) pero que podemos resumir en lo siguiente: la expansión de la ciudad de Zacatecas hacia Guadalupe ha inhibido el papel justo y equilibrado en los conflictos de la conurbación que pudiera cumplir la última. En lo urbano, el gobierno del estado, por medio de la Secretaría de Obras 
dad. Identificamos tres etapas a partir de que se llevó a cabo el primer proyecto de regeneración del $\mathrm{CHz}(1980)$.

La primera durante el lapso de 1980 a 1985, donde los montos destinados a su regeneración y embellecimiento son los más altos de la historia. En este periodo la característica del destino del presupuesto fue embellecer las calles, las fachadas y restaurar edificios abandonados (templos, teatros, casonas); a pesar del claro retraimiento de la actividad económica en la década de los ochenta en el país -que llevó a que se aplicaran políticas restrictivas en el gasto público- el proyecto de regeneración y rehabilitación del $\mathrm{CHz}$ tuvo su mejor momento (cuadro 1 ). El origen del financiamiento corrió a cargo exclusivamente del gobierno estatal, por medio del gasto programado en obra pública originado por los recursos extraordinarios provenientes del boom petrolero de 19801981. El objetivo de embellecer la ciudad fue dar los primeros pasos para atraer turistas y convertir esta actividad en el motor de desarrollo no sólo del $\mathrm{CHz}$, sino de la ciudad misma.

La segunda etapa fue de 1986 a 1996 y se caracterizó por una clara austeridad, pues sólo se ocupó en acciones de mantenimiento y conservación, pero no de regeneración. La mayor parte del presupuesto (principalmente del año 1993) se destinó a obras del cableado subterráneo y gastos de organización y representación de los evaluadores de la un Esco para declarar el Centro $\mathrm{H}$ istórico Patrimonio Cultural de la H umanidad. Los recursos se distribuyeron casi por igual entre los gobiernos federal (por medio de la Secretaría de Desarrollo Urbano y Ecología) y estatal (mediante su programa de obra estatal).

En 1996 se incrementa un poco el presupuesto y se destina sobre todo al pago de servicios profesionales, técnicos y especializados a agencias nacionales e internacionales para elaborar estrategias y proyectos de conservación solicitados por la un Esco y la recién creada Asociación de Ciudades Patrimonio. A partir de entonces, e inaugurando la tercera etapa, se aumenta de manera sustancial el presupuesto financiado principalmente por recursos de la unesco (2007), de la Asociación N acional de Ciudades M exicanas del Patrimonio M undial (ANCM PM, 2007) y de los gobiernos estatal y municipal. Además, la inversión privada aparece como un detonante importante de rehabilitación, pues en este periodo bancos (Banamex, Bancomer) y empresas de telecomu-

Públicas (y en menor medida el ayuntamiento de Zacatecas), tiene el control de la planeación y ejecución de acciones, según declaraciones hechas en entrevistas a funcionarios involucrados, dejando de lado al ayuntamiento de Guadalupe. 


\section{C uadro 1}

Presupuesto ejercido en el Centro $\mathrm{H}$ istórico de la ciudad de Z acatecas y su ritmo de crecimiento, 1981-2007 (en pesos constantes, 2002 $=100$ )

\begin{tabular}{crr}
\hline Año & Miles depesos & Incremento porcentual \\
\hline 1981 & $25,839.06$ & \\
1982 & $93,217.55$ & 360 \\
1983 & $59,901.46$ & -35.8 \\
1984 & $7,443.12$ & -88.6 \\
1985 & $3,304.48$ & -55.7 \\
1987 & 32.62 & -999.7 \\
1988 & 17.69 & -45.8 \\
1991 & 25.75 & 45.5 \\
1993 & 463.82 & 1800 \\
1994 & 23.36 & -95 \\
1995 & 0.98 & -95.8 \\
1996 & 38.61 & 39 \\
1997 & 191.2 & 495.1 \\
1998 & 123.05 & -35.7 \\
1999 & 286.09 & 232.5 \\
2000 & 39.67 & -86.3 \\
2001 & 92.44 & 235.3 \\
2002 & 242.94 & 262.7 \\
2005 & 140 & -42.4 \\
2006 & 53.02 & -62.2 \\
2007 & 541.59 & 102.1 \\
\hline
\end{tabular}

Fuente: Informes de gobierno y anexos de informes de gobierno, 1991-2007; presupuesto de egresos para el año fiscal 2007; Godezac (1981-2007) e índice de precios al consumidor (INEGI, 2007a).

nicaciones (Telmex, Televisa y TV Azteca) invierten en la restauración de museos, casonas, etc., por medio de sus fundaciones y fideicomisos para el fomento de la cultura, con lo que dan sus primeros pasos como empresas social mente responsables que ven la protección del patrimonio cultural como una forma de rentabilizar su capital. La característica de este nuevo periodo es nuevamente regenerar el $\mathrm{CHz}$, introducir nuevas áreas de atracción (museos, parques, templos, etc.) y mantener o conservar lo que ya se había rehabilitado.

Como se muestra en el cuadro 1, es muy evidente que los montos invertidos en el $\mathrm{cHz}$ en su primera etapa no se han superado. Sólo queda destacar que en 2007 el estado decidió situar al turismo cultural como la punta de lanza del desarrollo con la creación 
de un rubro financiero (autónomo) de fomento turístico cultural ${ }^{15}$ que, aun cuando promueve proyectos en otros sitios, ${ }^{16}$ el $\mathrm{CHz}$ sigue siendo el principal destino de inversiones y visitantes, por lo que las inversiones públicas se dispararon en ese año.

¿En qué consiste este proyecto? El proyecto actual de revitalizar el Centro $\mathrm{H}$ istórico no es un movimiento ciudadano para recuperar su ciudad, ni para reproducir sus prácticas sociales en este espacio, sino para transmutar la conciencia social ${ }^{17}$ de la población acerca de su derecho a un espacio público y a sus experiencias cotidianas para rentabilizar un espacio público. Dicho proyecto pretende hacer creer que el espacio remodelado es para su disfrute y goce incrementando las actividades de ocio (proliferación de antros, restaurantes, cafés) que atrae a jóvenes, turistas y pobladores de altos ingresos y despojando de espacios a actividades comerciales-artesanales tradicionales (venta de dulces, tamales, tunas) y a sus visitantes más comunes: familias de ingresos bajos (G amarra, 2005; H iernaux, 2005; Low, 2005). La idea de remodelar el CHz es el instrumento empleado para, por un lado, excluir a quienes no se sienten involucrados con la nueva imagen y, por el otro, atraer mayor número de visitantes que aprecian espacios modernos y bien cuidados que, en consecuencia, crean un efecto multiplicador para la actividad turística. La aceptación de esa imagen por parte de los zacatecanos es el reflejo de la pérdida de su identidad y cambio en su conciencia social que acepta, sin postura crítica, un espacio público que induce a la segregación y al uso privado.

Las principales acciones del proyecto de revitalización del casco central histórico son:

1. La inversión estatal en la remodelación estética de plazas y plazuelas con el fin de preservar el patrimonio cultural

15 Llamado "Zacatecas como destino turístico cultural" (Godezac, 2007: 13).

${ }^{16}$ El gobierno del estado de Zacatecas (Godezac, 2007a) lanzó el Programa de Desarrollo Regional, Innovación y Capital Humano (Proderic) para financiar proyectos que impulsen el desarrollo económico en todo el estado, pero es evidente, por lo menos en sus primeras tres versiones (2007-01, 2007-02 y 2008-01) que el turismo es el rubro más importante, pues es el que recibe la mayor parte del presupuesto y concentra el mayor número de proyectos.

${ }_{17}$ Cuando hablamos de transmutación de la conciencia social nos referimos a la acción que realiza un aparato con poder político para cambiar el sentido de la conciencia social sobre la diferencias entre clases sociales e intensificar su explotación. No creemos que el término ausencia de conciencia social sea el correcto, pues las clases sociales explotadas han demostrado en diferentes etapas históricas que sí cuentan con ella, sólo que en el momento actual está inhabilitada, inerte o dormida. 
colonial y en las cuales se ha retirado el comercio ambulante tradicional (venta de dulces o tunas, por ejemplo).

2. La restauración de edificios estratégicos con cada vez menor renta del suelo, obras que están a cargo de empresas de prestigio nacional: Sanborns, VIPS, entre otras. Aparentemente es una inversión privada, pero internamente está auspiciada por el gobierno estatal.

3. También está la tendencia contraria de abandono del mantenimiento de edificios públicos (iglesias, conventos, museos, teatros, casonas) que tienen poco valor turístico y muchos de los cuales albergan oficinas gubernamentales. Incluso hay zonas (barrios viejos) que se podrían aprovechar para aumentar la atracción y los recorridos turísticos, pero que en general requieren inversiones exorbitantes y cuya población mayoritaria es de ingresos medios y bajos (en la figura iv se muestran esas zonas potenciales).

4. La reestructuración de algunos edificios y lugares que no tienen ningún atractivo cultural ni turístico pero que formaban parte de la construcción del imaginario urbano y donde se reproducían ciertas prácticas cotidianas, con el objetivo de convertirlas en áreas rentables y atractivas para el ocio (Parque Sierra de Álica, Plaza 450, Plaza del Vergel).

5. La ruptura de la conciencia social del ciudadano con la construcción de complejos viales posmodernos en áreas que fueron creadas para fortalecer la identidad (la vieja Ciudad Universitaria o la antigua estación del ferrocarril).

6. Los complejos viales en los suburbios y zonas residenciales conjugados con obras de arte de escultores zacatecanos muestran, consciente 0 inconscientemente, que la ciudad se moderniza y que esas zonas son áreas chic, donde los pobladores de ingresos altos viven y se mueven diariamente (H idráulica-Campestre-Bernárdez).

7. Se trata de la creación de toda una política de promoción de imagen que fortalece la usurpación en dos niveles: el objetivo (promocionales hechos por el gobierno del estado para enaltecer el embellecimiento del Centro $\mathrm{H}$ istórico y el beneficio que traerá a la sociedad) y el subjetivo o subliminal (comentarios de boca en boca, reportajes en noticieros y programas de televisión y radio, opiniones de columnistas en prensa, entre otros). 
La propuesta de una ciudad turística manejada desde los años ochenta, y apoyada por un proyecto nacional (AN CM PM , 2007), se conjuga con la que el gobierno del estado clasificó como ciudad cultural y que se basa en la utilización del casco histórico en dos sentidos. Por un lado, instituir el imaginario de la preservación colonial como una inversión; crear la idea de lo nostál gico colonial sin respeto a la cultura e historia de la ciudad de Z acatecas y que permite actividades económicas lucrativas, por ejemplo, un automotor con casco de tranvía que, como tal, nunca existió en la ciudad. ${ }^{18}$ Y por otro, la usurpación de prácticas y actividades propias de los pobladores por parte del capital privado y del gobierno para hacerlas propias y rentabilizarlas: fiestas religiosas, ${ }^{19}$ populares, ${ }^{20}$ o cívicas, ${ }^{21}$ así como la creación de otras que nada tienen que ver con la idiosincrasia zacatecana. ${ }^{22}$

Ante este proceso, la actitud de los pobladores es de dos tipos. Por una parte, la pasiva y de presencia: disfrutan la imagen que proyectan los monumentos y realizan ${ }^{23}$ actividades de ocio. Es común que los habitantes (en especial los jóvenes) invadan las calles del Centro H istórico, sobre todo los fines de semana, los puentes y las vacaciones sin un objetivo específico, sólo pasear, pasarla bien y consumir los bienes y servicios que ofrece el casco histórico. En ese tenor, es posible clasificar a los pobladores de la ciudad en aquellos que sólo pasean y quienes pueden acceder a lo que produce la industria del ocio. Se trata de un proceso excluyente o elitista. Por la otra, se identifica la presencia de grupos aislados que se manifiestan por medio de asociaciones civiles o culturales para transmitir su visión de la ciudad y cómo quisieran que fuera. Entre ellos identificamos dos tipos: el primero más institucionalizado (en asociaciones civiles ${ }^{24}$ ) pero muy heterogéneo ${ }^{25}$ y cuyos puntos de vista algunas veces son críticos y otras superficiales de las acciones urbano-culturales estatales,

${ }^{18}$ En la ciudad de Zacatecas nunca existió un tranvía como medio de transporte urbano, sólo foráneo, hasta finales de los treinta, que la comunicaba con Villa de Guadalupe.

${ }^{19}$ Romerías del Patrocinio, Semana Santa, Q uema de Judas.

${ }^{20} \mathrm{M}$ orismas de Bracho.

${ }^{21}$ Procesión de la Fundación de la Ciudad y la Feria N acional de Zacatecas.

22 Festivales del Baile Folclórico, O toño M usical, Teatro en la Calle y Semana Santa en la Cultura.

${ }^{23} \mathrm{~N}$ os referimos, en términos marxistas, a la mercancía ocio. El consumidor como el puente final de la realización de mercancía y la obtención de una ganancia.

${ }_{24}$ Por ejemplo, el Grupo Chicomostoc y el Grupo Amigos del Parque Arroyo de la Plata.

${ }^{25}$ Compuesto de académicos e intelectuales, empresarios, comerciantes, funcionarios estatales, políticos, profesionistas, entre otros. 


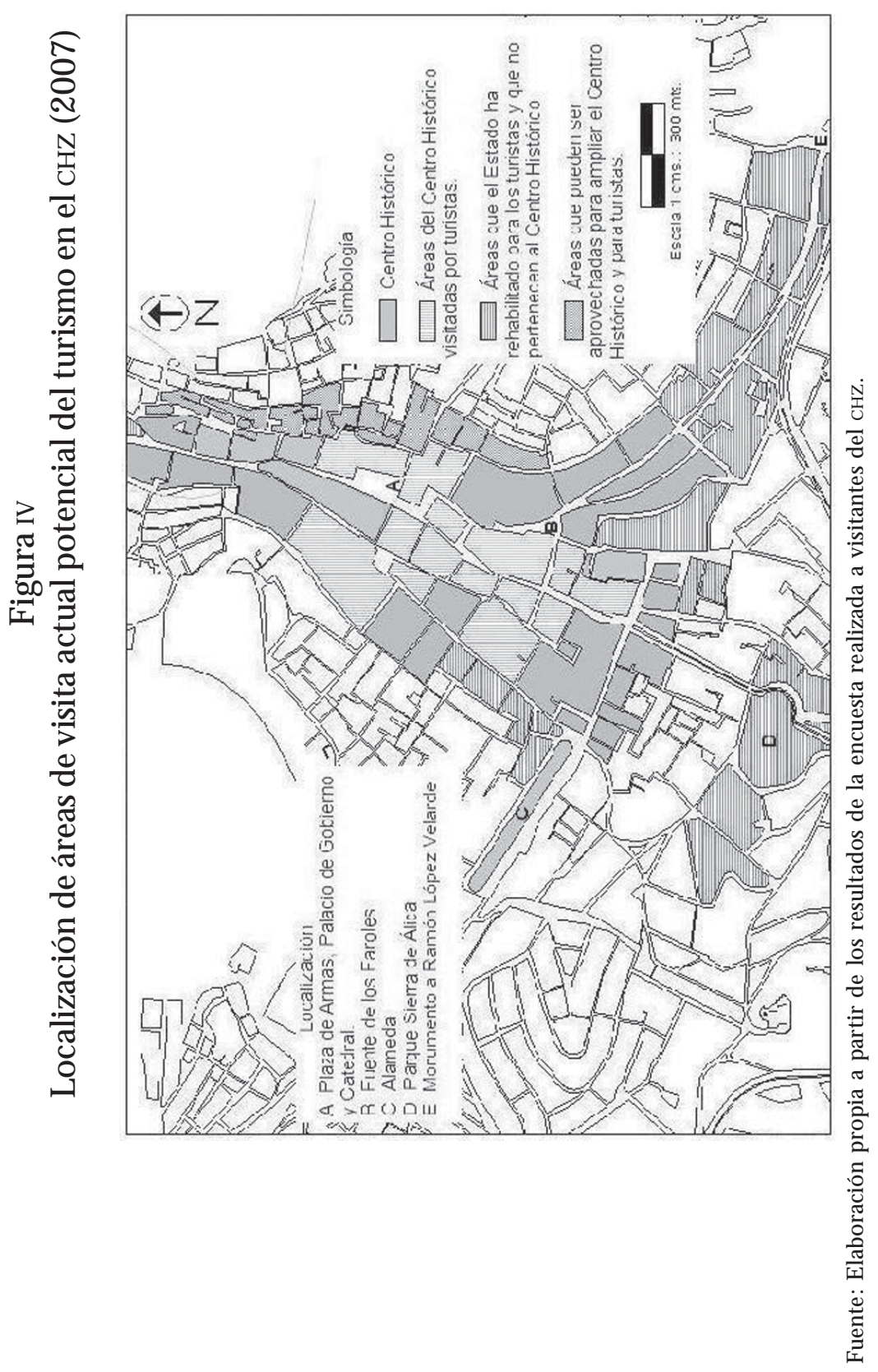


pero siempre respetando los principios de fomento al patrimonio colonial y la actividad económica establecida. El segundo es menos organizado y más homogéneo, ${ }^{26}$ pero mucho más crítico, pues aunque se enfoca en la política cultural estatal, busca un acceso equitativo a los recursos de la ciudad y evidencia las acciones fácticas de quienes no congenian con sus intereses.

\section{Importancia del turismo en la economía del cHz}

De acuerdo con los censos económicos del INEGI (1989, 1994, 1999, 2004), los servicios de hotelería y demás tipos de hospedaje, así como los de restaurantes, bares y centros nocturnos concentraron más de $34 \%$ del personal ocupado en servicios ofrecidos en el cHz durante 2004; sin embargo, su aportación en términos de productividad ingreso/trabajador deja mucho que desear. Las derramas económicas que en esas fechas dejaba el turista por hospedaje o alimentación en sitios localizados en el Centro H istórico eran relevantes, pero han disminuido de manera notable. Su mejor momento fue en 1999. En cambio, el resto de los servicios -que son los que demanda la población zacatecana- además de que son importantes en cuanto al ingreso promedio por trabajador (servicios profesionales, personales, financieros, médicos, odontológicos, veterinarios, de alquiler de equipo, bienes inmuebles, de esparcimiento o de transporte), han demostrado estabilidad, incluso algunas de esas ramas han crecido de manera importante (servicios profesionales, de asistencia o recreativos). En el cuadro 2 se muestra el ingreso promedio que obtuvieron los trabajadores de los establecimientos que ofrecen servicios localizados en el $\mathrm{CHz}$ en el periodo 1989-2004.

A pesar de los proyectos para convertir el $\mathrm{CHz}$ en un sitio de atracción turística, en la actualidad su economía dice lo contrario, pues continúa siendo un centro de comercios y servicios que demanda la población local (González, 2006) y los datos que aparecen en el cuadro 2 lo confirman. En 1999 las ramas de servicios al turista (hoteles, agencias de viajes, restaurantes y bares) mostraban un alto rendimiento ingreso/trabajador, pero en 2004 se evidencia un decaimiento y, por tanto, el renacimiento de las actividades económicas tradicionales.

Desde la perspectiva de afluencia de turistas, en el cuadro 3 se muestra el número de turistas que visitaron la ciudad anual-

${ }^{26}$ Cuenta con escultores, pintores, escritores, artesanos, etc. Todos de alguna forma están involucrados con la cultura popular zacatecana. 


\section{C uadro 2}

Promedio de ingresos derivados de la actividad/personal ocupado en las ramas de servicios del Centro H istórico 19892004 (en pesos constantes 2002=100)

\begin{tabular}{|c|c|c|c|c|}
\hline Rama/Año & 1989 & 1994 & 1999 & 2004 \\
\hline \multicolumn{5}{|l|}{ Servicios crediticios, bancarios } \\
\hline y auxiliares de crédito & 12.7 & & & 1 \\
\hline Servicios financieros & & & & 2.1 \\
\hline Aseguradoras y fianzas & & & & 0.4 \\
\hline Alquiler de bienes inmuebles & 2 & 1.1 & 1.4 & 0.9 \\
\hline \multicolumn{5}{|l|}{ O tros servicios inmobiliarios } \\
\hline (intermediación) & 0.2 & 4 & 1.5 & 1.1 \\
\hline \multicolumn{5}{|l|}{ Alquiler de equipo, } \\
\hline O tros servicios de alquiler & 1.1 & 1.1 & $\begin{array}{l}0.8 \\
0.6\end{array}$ & $\begin{array}{l}0.6 \\
1.6\end{array}$ \\
\hline Servicios educativos privados & 0.6 & 0.8 & 0.6 & 0.8 \\
\hline \multicolumn{5}{|l|}{ Servicios privados de investigación } \\
\hline \multicolumn{5}{|l|}{ Servicios privados médicos, } \\
\hline \multicolumn{5}{|l|}{$\begin{array}{l}\text { Servicios públicos médicos, } \\
\text { odontológicos y veterinarios }\end{array}$} \\
\hline $\begin{array}{l}\text { Servicios privados de asistencia social } \\
\text { Servicios de asistencia comercial, }\end{array}$ & & 0 & 0 & 0 \\
\hline $\begin{array}{l}\text { profesional y laboral } \\
\text { O rganizaciones políticas, civiles }\end{array}$ & 0.5 & 0.1 & 0 & 0.9 \\
\hline y religiosas & & & 0 & 0 \\
\hline \multicolumn{5}{|l|}{ Servicios de restaurantes, bares y } \\
\hline \multicolumn{5}{|l|}{ Servicios de hoteles y alojamiento } \\
\hline \multicolumn{5}{|l|}{ Servicios privados de esparcimiento } \\
\hline \multicolumn{5}{|l|}{ Centros privados recreativos, } \\
\hline \multicolumn{5}{|l|}{ Servicios profesionales, técnicos, } \\
\hline especializados y personales & 1.6 & 1.2 & 0.9 & 1.2 \\
\hline Servicios personales diversos & 0.4 & 0.7 & 0.5 & 0.6 \\
\hline Servicios de tintorería & 0.6 & 1.7 & 0.8 & 0.6 \\
\hline \multicolumn{5}{|l|}{ Servicios de reparación y } \\
\hline \multicolumn{5}{|l|}{ Servicios de reparación y } \\
\hline mantenimiento automotriz & 1 & 0.4 & 0.5 & 0.4 \\
\hline O tros servicios de reparación & 0.4 & 0.4 & 0.5 & 0.4 \\
\hline \multicolumn{5}{|l|}{ Servicios relacionados con } \\
\hline actividades agropecuarias & 0.5 & & & \\
\hline
\end{tabular}


continúa...

$\begin{array}{lllll}\text { Rama/Año } & 1989 & 1994 & 1999 & 2004\end{array}$

Servicios relacionados con

la construcción

Servicios relacionados

con transportes terrestres

Servicios relacionados con

instituciones financieras

Servicios de agencias de viaje,

mensajería y almacenaje

0.8

0.9

0.8

1.5

Fuente: Cálculos propios con base en censos económicos y en el índice de precios al consumidor (INEGI, 1989, 1994, 1999, 2004 y 2007a).

\section{C uadro 3}

Afluencia de turistas al Centro $\mathrm{H}$ istórico de la ciudad de Zacatecas y su ritmo de crecimiento (1988-2006)

\begin{tabular}{lrr}
\hline Año & Turistas & Incremento porcentual \\
\hline 1988 & 46,400 & \\
1990 & 322,613 & 6.95 \\
1991 & 371,313 & 1.5 \\
1992 & 400,790 & 7.9 \\
1993 & 555,457 & 38.5 \\
1994 & 341,024 & -38.7 \\
1995 & 372,688 & 9.2 \\
1996 & 392,590 & 5.3 \\
1997 & 554,041 & 41.1 \\
1998 & 694,946 & 25.4 \\
1999 & 584,386 & -16 \\
2000 & 612,417 & 4.7 \\
2001 & 665,000 & 8.5 \\
2003 & 698,586 & 5 \\
2006 & 748,842 & 7.1 \\
\hline
\end{tabular}

Fuente: Informes de gobierno y anexos de informes de gobierno (Godezac, 1988-2006).

mente de 1988 a 2006. La primera idea que expresa es su comportamiento errático: el más evidente es de 1988 a 1990. Y aunque la tendencia a largo plazo es aumentar dicha afluencia, ésta no se ha dado de manera espectacular. Incluso, Ilama la atención que la declaración del $\mathrm{CHz}$ como Patrimonio Cultural de la H umanidad en 1993 pareciera que tuvo un efecto negativo en la cantidad de turistas en los años siguientes, pues cayó en $61 \%$ y fue hasta cinco años después que recuperó el número de visitantes.

Incluso si analizamos la derrama económica que dejaron los turistas, los resultados tampoco son tan favorables para el sector 


\section{Cuadro 4 \\ Derrama económica dejada por los turistas y su ritmo de crecimiento 1998-2001 (en miles de pesos constantes, 2002 = 100)}

\begin{tabular}{lcr}
\hline Año & $\begin{array}{c}\text { Derrama económica } \\
\text { (miles depesos) }\end{array}$ & Diferencia porcentual \\
\hline 1988 & $3,193.26$ & \\
1989 & $3,408.05$ & 6.7 \\
1990 & $3,508.85$ & 2.9 \\
1991 & $3,337.45$ & -0.5 \\
1992 & $6,655.44$ & 99.4 \\
1993 & $6,744.23$ & 1.3 \\
1994 & $8,888.09$ & 31.7 \\
1995 & $6,144.34$ & -30.9 \\
1996 & $4,825.80$ & -21.5 \\
1998 & $6,037.18$ & 25.1 \\
1999 & $5,468.86$ & -9.5 \\
2000 & $5,474.35$ & 0 \\
2001 & $5,029.59$ & -8.2 \\
\hline
\end{tabular}

Fuente: Informes de gobierno y anexos de informes de gobierno (Godezac, 19882001) e índice de precios al consumidor (INEGI, 2007a).

turístico en la economía de la czG. En el cuadro 4 se señala, a precios constantes, que no ha habido un efecto económico importante y que los ritmos de crecimiento real mente son desdeñables. 0 lo que es peor, la afluencia de turistas ha sido más bien errática. Lo que significa que el turismo no ha crecido, y aunque así lo hiciera, no necesariamente se traduce en una derrama económica para la ciudad. Por tanto, y de acuerdo con estas cifras, el sector turístico realmente no parece ser la punta de lanza de la economía de la ciudad. En 2003 sólo el comercio al por menor de artículos para el cuidado de la salud (las farmacias) aportó a la economía del cHz la cantidad de 115'691,000 pesos, monto muy superior a cualquier año de la derrama económica turística (INEGI, 2004). Entonces, ¿por qué insistir en el proyecto de un $\mathrm{CHz}$ para turistas que gastan poco en comparación con otras actividades económicas?

\section{Efecto espacial del turismo en el $\mathrm{CHz}$}

A pesar de que este sector no ha mostrado un efecto en la economía urbana, la actividad turística como política se convierte en un elemento esencial para cumplir con los propósitos de rentabilidad del $\mathrm{CHz}$, lo cual se entiende dada la necesidad urgente de 
crear valor y empleos en la czG. Sin embargo, en las condiciones actuales el proyecto de revitalización del $\mathrm{CHz}$ contribuye a reforzar los efectos negativos: desvinculación con prácticas populares, exclusión social y transmutación de la conciencia social. Asimismo debemos apuntar que la actividad turística promueve la estructura monocéntrica de la czg y sus consecuencias en el deterioro del ingreso de buena parte de la población citadina.

Estudios realizados recientemente acerca de este tema nos permiten reforzar los argumentos planteados ${ }^{27}$ y exponer los referentes espaciales de la actividad económica del Centro $\mathrm{H}$ istórico que ayudan a explicar la conservación de su carácter monocéntrico.

El primer punto es el que se relaciona con la ubicación y extensión geográfica del CHz. La un Esco (1993: 34-35) definió espacialmente al $\mathrm{CHz}$ de forma muy diferente a la que aquí presentamos. ${ }^{28}$ En este estudio del $\mathrm{cHz}$ se determinan su extensión y límites por la percepción que tienen sus visitantes y pobladores, quienes emitieron opiniones sobre su área geográfica a partir de dos criterios: el primero que se identificó fue el de aglomeración, los entrevistados determinaron el espacio físico de concentración de actividades de todo tipo (económicas, culturales, sociales y políticas); y el segundo criterio se determinó por la estructura física, es decir, con base en la observación e identificación de los edificios que fijaron dónde comienza y termina el cHz. Después de analizar las encuestas y entrevistas se identificó que el cHz lo determina exclusivamente una columna vertebral de tres avenidas ( $\mathrm{H}$ idalgo, Tacuba-G uerrero y Genaro Codina-Fernando Villalpando de norte a sur, y de este a oeste las avenidas J uárez-H idalgo). En la figura ıv se observa la extensión del $\mathrm{CHz}$, de acuerdo con la opinión de pobladores y turistas. Los zacatecanos conciben la extensión del Centro H istó-

${ }^{27}$ Durante el periodo octubre-diciembre de 2007 efectuamos diversas actividades de campo para obtener información sobre la actividad turística en el cHz. Recibieron especial atención los aspectos económicos, simbólicos y de referenciación espacial de la actividad de los turistas y visitantes del CHz. Se efectuó la Encuesta a Visitantes del Centro Histórico de la ciudad de Zacatecas (con financiamiento Proderic 2007-01), compuesta por 250 entrevistas distribuidas por cuotas entre sus diferentes perfiles. No se presentan todos los resultados estadísticos considerando el objeto del presente artículo. Se realizaron distintas encuestas dirigidas a tres tipos de visitantes: 1) pobladores de la CZG, 2) turistas nacionales y extranjeros, y 3 ) visitantes y turistas con menos de un día disponible para recorrer la ciudad. La ubicación de los encuestados, los motivos y destinos de sus recorridos permitieron, junto con las entrevistas a personajes clave (funcionarios, académicos, etc.), definir algunas variables importantes para este análisis.

${ }^{28}$ El espacio físico comprende de norte a sur desde el Barrio de la Pinta y el ex Templo de M exicapan hasta la interconexión de las avenidas J uárez e H idalgo, anexando la parte vieja de las avenidas Rayón, M orelos y el boulevard López M ateos; y en dirección este a oeste desde el monumento a Ramón López Velarde a La Alameda (unesco, 1993). 
rico más pequeña que lo que determinó la un Esco, que no necesariamente contabiliza todo el patrimonio histórico y cultural de la ciudad, pues los habitantes generalmente asocian el $\mathrm{cHz}$ con la concentración de actividades en un espacio determinado que permite la interacción social y con los monumentos que mayor simbolismo tienen en la memoria colectiva.

Los pobladores de la czG están bastante de acuerdo con las inversiones realizadas en el $\mathrm{CHz}$. Para ellos es motivo de orgullo el estado de conservación de calles, edificios, monumentos y plazas. Existe una plena identificación con el proceso de revitalización, ya que consideran que beneficia a la ciudad en especial por su potencial turístico. Los pobladores entrevistados que visitan el CHz en su mayoría son adultos jóvenes y prácticamente a todos les agrada que haya turismo, sólo $16 \%$ opina lo contrario porque prefieren la tranquilidad, porque usurpan su cultura o porque se comercia la cultura zacatecana.

Para los habitantes de esta ciudad la razón principal por la que los turistas visitan el cHz es conocer sus edificios históricos, así como su herencia cultural y su tranquilidad. Los zacatecanos jamás asocian los eventos culturales y de entretenimiento organizados por el gobierno estatal con la llegada de turistas. De las entrevistas se desprende la opinión muy arraigada de que las actividades en el CHz son para generar beneficios económicos (aunque sea sólo para algunos): el turismo es la única manera que tiene la ciudad para salir adelante. ${ }^{29}$

Como es lógico, la delimitación física del $\mathrm{cHz}$ que expresan los turistas es aún más reducida, ya que sólo consideran como área histórico-turística la parte central de la zona determinada por los zacatecanos (14 manzanas de las 45 definidas por los pobladores) y muy por debajo de la que definió la unEsco (156 manzanas), que tiene a la avenida $\mathrm{H}$ idalgo como su referente. Esto tiene fuertes implicaciones en la economía zacatecana y en especial en la implementación de políticas de fomento al turismo y desarrollo económico ya que, en primer lugar, los limitados recorridos que realizan los turistas provocan una fuerte disputa por el uso de espacios comerciales (y su consecuente elevación de costos) y, en segundo lugar, porque al no visitar el resto del $\mathrm{CHz}$, el turista tiene pocas opciones de entretenimiento y reduce

${ }^{29}$ En palabras de un entrevistado: "Porque no hay más para la ciudad que el turismo". Sin embargo, al cuestionarlos sobre los efectos en el empleo consideran que la actividad turística no ha tenido ningún efecto en la creación de empleos. 
su estadía y montos de gasto en la ciudad. ${ }^{30}$ Así, esta reducida área de 14 manzanas se compone de negocios dedicados a la gastronomía (restaurantes, cenadurías, cafeterías, loncherías), venta de souvenirs (joyería, dulces y artesanías), de entretenimiento nocturno (antros, bares, cantinas), hospedaje (hoteles, hostales, moteles y casas de asistencia), arte y cultura (galerías, museos, librerías) y de prestación de servicios turísticos (agencias de viajes y recorridos).

El área definida y visitada por los turistas se caracteriza porque es visualmente atractiva (palacios, iglesias, casonas, calles, callejones, plazas y plazuelas de origen colonial con fuerte significado histórico), lo que promueve actividades de esparcimiento: recorridos y consumo de bienes y servicios tradicionales (souvenirs, gastronomía, hospedaje). Para el visitante, el atractivo de la arquitectura colonial es el principal motivo. El conocimiento de aspectos históricos y simbólicos sólo es interesante para muy pocos visitantes. Tampoco son motivo de visita los servicios, comercio o entretenimiento que pueda proporcionar el $\mathrm{CHz}$, es más, la escasez y calidad de estos son limitantes para incrementar la estancia en la ciudad. El consumo turístico principal es el alojamiento, del cual se han beneficiado las cadenas hoteleras y, en menor medida pero de manera creciente, pequeños hoteles y hostales de empresarios locales. O tro sector beneficiado es el restaurantero, aunque las evaluaciones sobre la calidad y precio de estos servicios son divergentes. El uso de otros servicios turísticos es limitado, salvo por las compras en tiendas de conveniencia. ${ }^{31}$

Debido a los cortos recorridos realizados por los turistas en los últimos años (1998-2008), se implementaron políticas de conservación, remodelación y difusión de lugares situados fuera de esta zona turística básica con la intención de aumentar y mejorar la oferta (figura iv). Es conocido que muchas de ellas no tienen el valor histórico ni simbólico de los espacios y edificaciones ca-

${ }^{30}$ De acuerdo con el Godezac (1999), el promedio que permanece un turista en la cGz es de 1.6 días; según un ex secretario de Turismo (entrevista realizada en diciembre de 2007), en 2004 el promedio aumentó a dos días; y los datos de nuestra encuesta dan un resultado de 2.8 días.

${ }^{31}$ Es bastante complicado generalizar, pues la utilización de servicios depende fuertemente del tiempo de estadía, edad, origen e interés, pero podemos afirmar que sólo un tercio de los entrevistados utilizó los recorridos turísticos, de los cuales se quejaron por la calidad de la información ofrecida, lo reducido del recorrido y el costo excesivo. Los antros, bares y cantinas los frecuentaron exclusivamente jóvenes que también se quejaron de la calidad del servicio y su costo. Sólo un tercio de los turistas visitó los múltiples museos que ofrece el cHz. 
racterísticos del $\mathrm{CHz}$, pero se les considera con potencial de atracción. El éxito ha sido relativo, las encuestas realizadas a turistas indican que no visitan ni reconocen como parte del atractivo turístico de la ciudad áreas como el Parque Sierra de Álica o el monumento al poeta López Velarde en la vieja Ciudad Universitaria. En realidad estos espacios, en especial el primero, los aprovechan los pobladores locales y los visitantes de corta estancia de la región.

Al entrevistar a los visitantes con menos de un día para recorrer la ciudad, fue posible identificar entre ellos a un segmento cuyos patrones de visita son por demás interesantes para el sostenimiento del monocentrismo de la czG. A estos no se les ha dado la atención debida y es posible que incluso compitan en importancia con los turistas tradicionales. Sus prácticas de consumo son significativas no sólo por sus montos, también por el efecto económico que provocan en los diferentes sectores de la ciudad. Es el tipo de visitante que llega a la ciudad con motivos muy específicos y tiempo limitado; tiene la característica de que viene de lugares muy cercanos a la capital (generalmente de la misma región o del propio estado) a realizar actividades de compras, entretenimiento o trámites burocráticos. ${ }^{32}$ Debido a su frecuencia de viaje (visitan la ciudad en promedio tres veces al año), es bastante observador y crítico de la oferta del $\mathrm{CHz} ;{ }^{33}$ por otro lado, su radio de acción es mucho más amplio que el del turista clásico, pues recorren las áreas que son el motivo principal de su visita: compras de celulares, víveres, ropa, juguetes, etc. Este tipo de visitante regional circula por las áreas este, sur y sureste del $\mathrm{CHZ}^{34}$ (figura IV). Además de los motivos específicos que lo llevaron a visitar la ciudad, realiza (aunque en menor grado) actividades turísticas tradicionales: visita lugares de atracción, compra souvernirs, asiste a eventos de entretenimiento y utiliza servicios turísticos. Su efecto económico se debe determinar pues complementa el consumo local y fortalece la rentabilidad de los establecimientos comerciales y de servicios dedicados a la demanda local. Aun cuando no existen datos duros al respecto, las

${ }^{32}$ De acuerdo con nuestras encuestas, este tipo de visitante provenía de municipios cercanos a la czG: Guadalupe, M orelos, Panuco y Villanueva.

${ }^{33}$ La mayor parte de ellos se quejaron del servicio turístico, en especial del que ofrece el transporte público (taxis y camiones) y de los eventos de entretenimiento. A pesar de que califican al $\mathrm{cHz}$ como muy bonito y que constantemente lo encuentran remozado, consideran que la oferta de servicios es pobre, de mala calidad y/o de costo excesivo.

${ }_{34}$ Parque Independencia, Plazuela de Vivac, Plazuela Genaro Codina, avenidas Rayón, Juárez, Torreón, Aldama, Arroyo de la Plata, Guerrero, Primero de M ayo y López Velarde. 
entrevistas realizadas a algunos comerciantes permiten suponer que existen negocios en el $\mathrm{CHz}$ que dependen del consumo de este segmento de visitantes, por lo menos debemos suponer que sus adquisiciones de bienes y servicios complementan los que realizan los pobladores de la ciudad, reforzando las economías de escala, alcance y aglomeración que permite la concentración de la actividad productiva en el $\mathrm{CHz}$.

Por tanto, se puede afirmar que cada grupo social que visita el $\mathrm{CHz}$ tiene conceptos distintos acerca de éste: la población residente lo concibe de manera más integral mediante consideraciones económicas, históricas y físicas. En cambio el turista lo identifica más con edificios coloniales y los sitios de esparcimiento. Ambas concepciones permiten ubicar áreas específicas de apropiación y uso del espacio urbano. En el $\mathrm{CHz}$ existen por lo menos dos zonas distintas de aprovechamiento turístico integradas a un área más amplia identificada por los pobladores de la ciudad. Por otro lado, las percepciones de los visitantes y pobladores sobre el significado y función del $\mathrm{CHz}$ tienden a coincidir con la idea de su potencial explotación económica, más que en su valor histórico y cultural.

Lo anterior coincide con algunos análisis teóricos como el de Troncoso y Almirón (2005), quienes opinan que el patrimonio histórico lo determinan los procesos sociales que expresan conflictos de intereses entre grupos y que le dan simbolismo a un conjunto de bienes tangibles e intangibles, modificando su significando y su inventario con el paso del tiempo. Sin embargo, dicha resignificación adquiere mayor importancia cuando alcanza utilidad y valor por los visitantes. El patrimonio adquiere un vaIor comercial que, por un lado, induce una actividad económica redituable (en muchos casos se vuelve la panacea para disminuir la pobreza o el estancamiento económico) y, por el otro, se hace atractivo con base en un conjunto de ideas, imaginarios y percepciones que se crean los turistas -incluso desde sus lugares de origen- y que pueden variar dependiendo de su práctica turística y la satisfacción que les proporcione el inventario patrimonial.

Sin embargo, es el Estado o los poderes políticos legalmente constituidos quienes llevan a cabo el proceso de selección patrimonial, a pesar de que es la sociedad quien pueda proponer y sustraer sus representaciones. La selección del patrimonio como valor universal u homogéneo no siempre lo comparten los distintos grupos sociales o sectores de la comunidad, de manera que el espacio determinado como patrimonio se considera un lugar de 
conflicto, tensión, lucha y negociación entre grupos que se miden de acuerdo con sus relaciones de poder (Troncoso y Almirón, 2005, 61).

\section{Conclusiones}

Q uizás la idea de etapas de expansión y crecimiento urbano que tuvo validez hace décadas, hoy se deba evaluar otra vez. Las ciudades que tienen un papel mundial poco relevante no sólo presentan estados de subdesarrollo económico y social, también padecen un estado tardío de expansión y de regeneración de la estructura urbana. En ciudades donde la relación capital-trabajo se manifiesta en el dominio de actividades terciarias de relevancia exclusivamente local, las etapas de expansión y reestructuración urbana se retrasan y traslapan intensificando las contradicciones inherentes.

El proceso de suburbanización se entremezcla con un retorno a la ciudad, en especial un regreso a hacer del Centro H istórico el lugar por excelencia de la actividad económica urbana en beneficio de quienes tienen mayor poder adquisitivo. La estructura de ciudades como Zacatecas-Guadalupe, a finales del siglo $x x$ y comienzos del $x x$, vive un proceso de suburbanización lento y auspiciado sobre todo por los grupos de ingreso alto que se conjuga con un proceso de reestructuración económica interna que aún no puede consolidar la actividad turística y donde los principios de localización de negocios es inestable. La población ha tenido que adaptarse a esos cambios.

Tal vez algunas ciudades se han transformado de excluyentes a participativas (Rossi, 2003; Carrión, 2005) planteando que la revitalización de los centros históricos ha permitido revalorar los imaginarios urbanos y las prácticas colectivas que se traducen en una mayor participación ciudadana en las decisiones urbanas; pero en las ciudades subdesarrolladas, por el contrario, el capital y la gestión estatal han intensificado su voraz política neoliberal de reestructuración del espacio urbano, aunque la innovación ahora es utilizar la identidad urbana y los imaginarios del patrimonio cultural colonial como los instrumentos que permiten transmutar la conciencia de las clases sociales hacia la inercia, por un lado, y excluir a quienes no pueden acceder a la industria del ocio, por el otro. La centralidad sigue teniendo un fin económico. 
La revitalización del $\mathrm{CHz}$, efectuada a partir de expectativas de explotación turística, es un elemento que ha reforzado el carácter monocéntrico de la estructura urbana de la cGz. Las encuestas realizadas perfilan referentes espaciales distintos para el CHz que implican áreas de intercambio económico diferenciadas por el tipo de visitante. La política gubernamental ha privilegiado la dotación de servicios a los turistas tradicionales y sus espacios de disfrute dejando de lado la atención y el fomento de servicios para visitantes de características distintas, como los que aquí denominados visitantes regionales. Las áreas que frecuenta este grupo no han sido receptoras de mejoras urbanas sustanciales y constituyen una zona de oportunidad para las políticas de mantenimiento urbano. Las demandas de los visitantes regionales contribuyen a la actividad económica local en dimensiones no estudiadas, pero que pueden ser significativas.

Por otro lado, las percepciones so bre la funcionalidad del $\mathrm{CHZ}$ acerca de la generación de beneficios económicos han ganado terreno en la población residente, en tanto que para los turistas el valor principal de estos espacios está en su estética, omitiendo su valor como elementos simbólicos de un pueblo con herencia histórica. La transmutación de la conciencia social es cada vez más evidente. Actualmente, las reivindicaciones y protestas sociales existen en el espacio urbano zacatecano, pero el Centro $\mathrm{H}$ istórico ya no se concibe como el escenario de esas manifestaciones, pues los imaginarios sociales lo consideran el lugar idóneo para rentabilizar el ocio.

De acuerdo con la aquí expuesto, es necesario reformular el proyecto para el cHz que integre no sólo los intereses relacionados con la producción económica, también que sea respetuoso de los valores culturales que representa y que permite recrear.

\section{Bibliografía}

Alegría, Tito (1994), "Condiciones espaciales de la pobreza urbana y una propuesta para su disminución", Frontera Norte, 1, núm. esp., El Colegio de la Frontera N orte, Tijuana, pp. 61-76.

Alegría, Tito (1994a), “Consideraciones teóricas y metodológicas de estructura urbana", Reporte de Investigación, El Colegio de la Frontera $\mathrm{N}$ orte, Tijuana. 
AN CM PM (2007), Financiamiento, Asociación N acional de Ciudades $M$ exicanas del Patrimonio M undial, A. C., M éxico, http://ww w.ciudadesmexicanaspatrimonio.org/ index_fla.htm.

Carrión, Fernando (2005), “El Centro H istórico como proyecto y objeto de deseo", Eure, xxx। (93), Santiago, pp. 89-100.

Delgado, Raúl, Víctor Figueroa y M argarita H offner (1991), Zacatecas. Sociedad, economía, política, cultura, Universidad N acional Autónoma de M éxico, M éxico.

Esparza, M iguel (1996), Elementos básicos; la estructura económica y el cambio regional en Zacatecas durante la década de los ochenta, Universidad Autónoma de Zacatecas, Zacatecas.

Figueroa, Víctor (1986), Reinterpretando el subdesarrollo. Trabajo general, clase y fuerza productiva en América Latina, Siglo XXI, M éxico.

Gamarra, Garikoitz (2005), "Ciudad, poder, identidad. Bilbao: pasión y muerte de lo urbano", Bifurcaciones, 2, Santiago, pp. 1-11.

Godezac (1986), Zacatecas, 6 años de gobierno 1980-1986, José Guadalupe Cervantes Corona, Zacatecas.

Godezac (1987), Primer Informe de Gobierno, Genaro Borrego Estrada, Zacatecas.

Godezac (1987a), Anexo, acciones y estadísticas. Desarrollo social, Primer Informe de Gobierno, Genaro Borrego Estrada, Zacatecas.

Godezac (1987b), Anexo, acciones y estadísticas. Desarrollo económico, Primer Informe de Gobierno, Genaro Borrego Estrada, Zacatecas.

Godezac (1988), Segundo Informe de Gobierno, Genaro Borrego Estrada, Zacatecas. 
Godezac (1988a), Anexo estadístico III. Desarrollo económico, Segundo Informe de Gobierno, Genaro Borrego Estrada, Zacatecas.

Godezac (1988b), Anexo estadístico IV. Infraestructura para el desarrollo, Segundo Informe de G obierno, G enaro Borrego Estrada, Zacatecas.

Godezac (1989) Tercer Informe de Gobierno, Genaro Borrego Estrada, Zacatecas.

Godezac (1989a), Anexo estadístico III. Desarrollo económico, Tercer Informe de Gobierno, Genaro Borrego Estrada, Zacatecas.

Godezac (1990), Cuarto Informe de G obierno, Genaro Borrego Estrada, Zacatecas.

Godezac (1990a), Anexo estadístico III. Desarrollo económico, Cuarto Informe de Gobierno, Genaro Borrego Estrada, Zacatecas.

Godezac (1991), Q uinto Informe de Gobierno, Genaro Borrego Estrada, Zacatecas.

Godezac (1991a), Anexo Estadístico III. Desarrollo económico, Quinto Informe de Gobierno, Genaro Borrego Estrada, Zacatecas.

Godezac (1991b), Anexo estadístico IV. Infraestructura para el desarrollo, Quinto Informe de Gobierno, Genaro Borrego Estrada, Zacatecas.

Godezac (1992), Sexto Informe de G obierno, Pedro de León Sánchez, Zacatecas.

Godezac (1992a), Anexo estadístico III. Desarrollo económico, Sexto Informe de Gobierno, Pedro de León Sánchez, Z acatecas.

Godezac (1993), Primer Informe de Gobierno, Arturo Romo Gutiérrez, Zacatecas. 
Godezac (1993a), Tomo III. Desarrollo económico, Primer Informe de Gobierno, Arturo Romo Gutiérrez, Zacatecas.

Godezac (1993b), Tomo IV. Desarrollo Social, Primer Informe de Gobierno, Arturo Romo Gutiérrez, Zacatecas.

Godezac (1994), Segundo Informe de Gobierno, Arturo Romo Gutiérrez, Z acatecas.

Godezac, 1994a, Anexo III. Desarrollo económico, Segundo Informe de Gobierno, Arturo Romo Gutiérrez, Zacatecas.

Godezac (1994b), Anexo IV. Desarrollo social, Segundo Informe de Gobierno, Arturo Romo Gutiérrez, Zacatecas.

Godezac (1995), Tercer Informe de Gobierno, Arturo Romo Gutiérrez, Z acatecas.

Godezac (1995a), Anexo III. Desarrollo económico, Tercer Informe de Gobierno, Arturo Romo Gutiérrez, Zacatecas.

Godezac (1995b), Anexo IV. D esarrollo social, Tercer Informe de Gobierno, Arturo Romo Gutiérrez, Zacatecas.

Godezac (1996), Cuarto Informe de Gobierno, Arturo Romo Gutiérrez, Z acatecas.

Godezac (1996a), Tomo III. Desarrollo económico, Cuarto Informe de Gobierno, Arturo Romo Gutiérrez, Zacatecas.

Godezac (1996b), Tomo IV. Desarrollo social, Cuarto Informe de Gobierno, Arturo Romo Gutiérrez, Zacatecas.

Godezac (1997), Quinto Informe de Gobierno, Arturo Romo Gutiérrez, Z acatecas, M éxico.

Godezac (1997a), Tomo III. Desarrollo económico, Quinto Informe de Gobierno, Arturo Romo Gutiérrez, Zacatecas.

Godezac (1997b), Tomo IV. Desarrollo social, Quinto Informe de Gobierno, Arturo Romo Gutiérrez, Zacatecas. 
Godezac (1998), Sexto Informe de G obierno, Arturo R omo G utiérrez, Zacatecas.

Godezac (1998a), Tomo III. Desarrollo económico, Sexto Informe de Gobierno, Arturo Romo Gutiérrez, Zacatecas.

Godezac (1998b), Tomo IV. Desarrollo económico, Sexto Informe de Gobierno, Arturo Romo Gutiérrez, Zacatecas.

Godezac (1999), Primer Informe de Gobierno, Ricardo M onreal Ávila, Zacatecas.

Godezac (1999), Anexo IV. Desarrollo social, vol. III, Primer Informe de Gobierno, Ricardo M onreal Ávila, Zacatecas.

Godezac (1999), Anexo IV. Desarrollo social, vol. V, Secretaría de D esarrollo Social, septiembre-diciembre 1998, Primer Informe de G obierno, Ricardo M onreal Ávila, Zacatecas.

Godezac (1999), Anexo II. Desarrollo económico, vol. I, Secretaría de Turismo, enero-septiembre 1999, Primer Informe de Gobierno, Ricardo M onreal Ávila, Zacatecas.

Godezac (1999), Anexo II. Desarrollo económico, vol. II, Secretaría de O bras Públicas, enero-septiembre 1999, Primer Informe de G obierno, Ricardo M onreal Ávila, Z acatecas.

Godezac (2000), Segundo Informe de Gobierno, Ricardo M onreal Ávila, Zacatecas.

Godezac (2000), Tomo III. Desarrollo económico, Segundo Informe de Gobierno 1999-2000, Ricardo M onreal Ávila, Zacatecas.

Godezac (2001), Tercer Informe de G obierno, Ricardo M onreal Ávila, Zacatecas.

Godezac (2002), Cuarto Informe de Gobierno, Ricardo M onreal Ávila, Zacatecas.

Godezac (2003), Quinto Informe de Gobierno, Ricardo M onreal Ávila, Zacatecas. 
Godezac (2005), Primer Informe de Gobierno, Amalia García M edina, Zacatecas.

Godezac (2005a) Tomo II. Hacia un desarrollo económico sustentable, Primer Informe de Gobierno, Amalia García M edina, Zacatecas.

Godezac (2006), Cuenta pública. Ejercicio 2005, Ingresos, Secretaría de Finanzas, Zacatecas, www.zacatecas.gob.mx/ CuentaPublica/2005/ingresos.pdf.

Godezac (2006a), Segundo Informe de Gobierno, Amalia García M edina, Zacatecas.

Godezac (2006b), Tomo II. H acia un desarrollo económico sustentable, Segundo Informe de Gobierno, Amalia García M edina, Zacatecas.

Godezac (2007), Decreto no. 430, Presupuesto de egresos del estado para ejercicio fiscal del año 2007, Periódico O ficial del Gobierno del Estado de Zacatecas, tomo CX VI, núm. 104, Suplemento, 30 de diciembre de 2006, Zacatecas.

Godezac (2007a), Programa de Desarrollo R egional, Innovación y Capital H umano (Proderic), Secretaría de Planeación y Desarrollo Regional, Zacatecas.

González, Guadalupe, 2004, “D el monocentrismo al policentrismo intraurbano: un modelo explicativo del cambio en la estructura de centros en la zona conurbada ZacatecasGuadalupe (1990-2000)", tesis doctoral, El Colegio de la Frontera N orte, Tijuana.

González, Guadalupe (2006), "Segregación socioespacial en la zona conurbada Zacatecas-G uadalupe (1990-2000), Boletín de los sistemas Nacionales Estadístico y de información geográfica, 2 (2), IN EGI, M éxico, pp. 79-94.

G onzález, Guadalupe (2006a), “R eforzamiento de una estructura monocéntrica: el Centro H istórico de la ciudad de Zacatecas (1990-2003)", Revista Electrónica Zacatecana sobre 
Población y Sociedad, tercera época, año 6, 27, Universidad Autónoma de Zacatecas, Zacatecas, pp. 1-23.

González, Guadalupe (2006b), "Revitalización del Centro Histórico de la ciudad de Zacatecas: exclusión y transmutación de la conciencia social", en José Gasca (coord.), La construcción de perspectivas de desarrollo en México desde sus regiones, $\mathrm{M}$ emoria del $11^{\circ}$ Encuentro $\mathrm{N}$ acional sobre Desarrollo Regional en M éxico, Amecider, M érida, pp. 1-19.

Gordon, Peter y H arry Richardson (1995), “Employment Decentralization in U. S. M etro Areas: is Los Angeles an outlier or the norm?", Working Paper, Lusk Center Research Institute, UCLA, Los Ángeles.

Gordon, Peter y H arry Richardson (1995a), "Travel Time Distributions in a Dispersed M etropolis", Working Paper, School of Urban and Regional Planning, UCLA, Los Ángeles.

Gordon, Peter y H arry Richardson (1996), "Beyond Polycentricity: The Dispersed M etropolis, Los Angeles 1970-1990", Journal of the American Planning Association, 62 (3), UCLA, Los Ángeles, pp. 289-295.

H iernaux, Daniel (2005), “Imaginarios y lugares en la reconquista de los centros históricos", Ciudades, 65, Puebla, pp. 15-21.

INEGI (1989), Censos económicos 1989, Aguascalientes, disco compacto.

INEGI (1992), XI Censo General de Población y Vivienda 1990, Zacatecas, Aguascalientes, disco compacto.

INEGI (1994), Censos económicos 1994, Aguascalientes, disco compacto.

INEGI (1996), Conteo 95, Resultados definitivos. Tabulados básicos, Aguascalientes, disco compacto.

INEGI (1999), Censos económicos 1999, Aguascalientes, disco compacto. 
INEGI (2000), XII Censo General de Población y Vivienda 2000. Sistema para la consulta de Información Censal (Scince), Aguascalientes, disco compacto.

INEGI (2004), Censos económicos 2004, A guascalientes, disco compacto.

INEGI (2005), Encuesta Nacional de Empleo, Aguascalientes, Sistema de Consulta en línea, http://www.inegi.gob.mx/est/ contenidos/espanol/sistemas/ene/ene_trim/datos/ 2004trim4/03/03/2006.

INEGI (2006), Encuesta N acional de Empleo Urbano, Aguascalientes, Banco de Información Económica, Sistema de Consulta en línea, http://dgcnesyp.inegi.gob.mx/cgi-win/ bdieintsi.exe/Consultar/25/09/2006.

INEGI (2006a), II Conteo de Población y Vivienda 2005, Zacatecas. Resultados definitivos. Tabulados básicos. Aguascalientes, disco compacto.

INEGI (2007a), Índice Nacional de Precios al Consumidor (Mensual) 2002=100, Banco de Información Económica, Sistema de Consulta en Línea, http://dgcnesyp.inegi.gob.mx/ cgi-win/bdieintsi.exe/C onsultar.

Jaramillo, Samuel (1997), “El papel del mercado del suelo en la configuración de las ciudades latinoamericanas", Lincoln Institute of Land Policy, Reporte de Investigación, UCLA, Los Ángeles.

Kotkin, J oel (1999), "T he future of the Center: The C ore City in the N ew Economy", Policy Study no. 264, Reason Public Policy Institute, Boston.

Low, Setha (2005), "Transformaciones del espacio público en la ciudad latinoamericana: cambios espaciales y prácticas sociales", Bifurcaciones, 5, Santiago, pp. 1-14.

O rtiz, Anna (2005), “Renovación social y transformación urbanística: el barrio de Raval, Barcelona, Ciudades, 65, Puebla, pp. 54-59. 
O 'Sullivan, Arthur (1996), Urban Economics, Irvin-M cG raw H ill, Boston.

Richardson, H arry y Peter Gordon (1994), "N ew Data and Old M odels in Urban Economics", Working Paper, Lincoln Institute of Land Policy, UCLA, Los Ángeles.

Rossi, Ugo (2003), "N ápoles, ciudad democrática: la construcción del Centro H istórico como un espacio público", Economía, Sociedad y Territorio, Iv (13), El Colegio M exiquense, Toluca, pp. 1-26.

Staley, Samuel (2004), Can Downtows survive the 21th Century?, Reason Public Policy Institute, Boston.

Thuillier, Guy (2005), “El impacto socioespacial de las urbanizaciones cerradas: el caso de la región metropolitana de Buenos Aires", Eure, xxxı (93), Santiago, pp. 5-20.

Troncoso, Claudia y Analía Almirón (2005), "Turismo y patrimonio. H acia una relectura de sus relaciones", Aportes y Transferencias 1 (9), Universidad $\mathrm{N}$ acional del M ar de Plata, M ar de Plata, pp. 56-74.

Un ESCO (1993), Advisory Body Evaluation, World Heritage List no. 676, unESCO, París, http://whc.unesco.org/archive/ advisory_body_evaluation/676.pdf.

UnESCO (2007), Financial Regulations for the World Heritage Fund, UN ESCO, París, http://whc.unesco.org/en/financial regulations.

Recibido: 13 de octubre de 2006.

Reenviado: 28 de julio de 2008. Aceptado: 1 de agosto de 2008.

G uadalupe M argarita G onzález H ernández. Es doctora en ciencias sociales por El Colegio de la Frontera N orte, A. c.; licenciada y maestra en economía por la Universidad Autónoma de Zacatecas (UAZ). Actual mente es docente investigadora de la Unidad Académica de Ciencia Política de la UAz. Es profesora con perfil deseable en el Programa de M ejoramiento del Profesorado (Promep) y 
candidata a investigador en el Sistema $\mathrm{N}$ acional de Investigadores (SNI). Su línea de investigación actual es: procesos económicos, sociales y políticos en las ciudades mexicanas. Entre sus publicaciones destacan: "Segregación socioespacial, integración al mercado de trabajo y deterioro de los grupos de ingreso medio en la zona conurbada Z acatecas-G uadalupe, M éxico 2000", Papeles de Población, año 11, 46, Universidad Autónoma del Estado de M éxico, Toluca, pp. 79-108 (2005); "Segregación socioespacial en la zona conurbada Zacatecas-Guadalupe (1990-2000)", Boletín de Ios Sistemas Nacionales Estadístico y de Información Geográfica, 2 (2), INEGI, Aguascalientes, M éxico, pp. 79-94 (2006); en coautoría, Reproducción precaria familiar. Conceptualización y evidencias en Zacatecas-G uadalupe (1990-2004), Fomix-C onacyt-G odezac-Universidad Autónoma de Zacatecas, Zacatecas (2007); "M ercado de trabajo en la zona conurbada Zacatecas-G uadalupe, 2000: realidades y disyuntivas", en Silvana Figueroa (comp.), Economía, trabajo y educación en Zacatecas. Temas de interés actual, Gobierno del Estado de Zacatecas-Universidad Autónoma de Zacatecas, Zacatecas, pp.113-136 (2007). 